\title{
Bioinformatic analysis of gene regulation in the metal-reducing bacterial family Geobacteraceae
}

\author{
Julia Krushkal ${ }^{*}$, Ronald M Adkins², Yanhua Qu ${ }^{1}$, Jeanette Peeples ${ }^{2}$, Sreedhar Sontineni ${ }^{1,3}$, Ching Leang ${ }^{4}$, \\ Peter Brown ${ }^{4}$, Nelson D Young ${ }^{4}$, Toshiyuki Ueki ${ }^{4}$, Katy Juarez ${ }^{5}$, Derek R Lovley ${ }^{4}$ \\ From UT-ORNL-KBRIN Bioinformatics Summit 2010 \\ Cadiz, KY, USA. 19-21 March 2010
}

\section{Background}

Knowledge of how structural genome differences among microorganisms lead to variation in gene regulation is fundamentally important for our understanding of the functioning of gene regulatory pathways and their individual components. This knowledge is also necessary for our better understanding of the genomic changes leading to adaptation to diverse and changing environments. Our research focuses on Geobacteraceae, a metal-reducing family of delta-Proteobacteria, which are capable of harvesting electricity from organic matter and environmental bioremediation of organic and metal pollutants. We are investigating molecular mechanisms which allow these species to adapt and regulate their responses to environmental stimuli, which result in energy production and removal of environmental pollutants.

\section{Materials and methods}

In this presentation, we describe computational analysis of gene regulation in multiple species from this family. Our ongoing analyses include cataloguing and integrating information about gene regulation, in silico prediction of transcription factor binding sites, and investigation of functional effects of genome-scale and single nucleotide and amino acid level changes in transcription factors, operons, and regulatory sequence elements in Geobacteraceae. Specifically, we discuss our computational pipeline for comparative analyses of variation in regulatory organization among species and strains of Geobacteraceae, which allows us to investigate changes in operon organization and to track the effects of operon rearrangements and individual single nucleotide base pair level changes on regulatory sequence elements. We also describe our computational analyses of two systems of gene expression, one of gene regulation by the PilR enhancer binding protein and another by the TetR family of transcriptional regulators. Finally, we describe our ongoing efforts to catalog available information on gene regulation and operon organization of Geobacter sulfurreducens, a model representative of the Geobacteraceae family.

\section{Acknowledgements}

This research was supported by the Office of Science (BER), U.S. Department of Energy, Cooperative Agreement No. DE-FC02-02ER63446.

\section{Author details}

${ }^{1}$ Department of Preventive Medicine, University of Tennessee Health Science Center, Memphis, TN 38163, USA. ${ }^{2}$ Department of Pediatrics, University of Tennessee Health Science Center, Memphis, TN 38163, USA. ${ }^{3}$ Program in Bioinformatics, University of Memphis, Memphis, TN 38111, USA.

${ }^{4}$ Department of Microbiology, University of Massachusetts, Amherst, MA 01003, USA. ${ }^{5}$ Departamento de Ingeniería Celular y Biocatálisis, Instituto de Biotecnología, Universidad Nacional Autónoma de México, Cuernavaca 62271, México.

Published: 23 July 2010

doi:10.1186/1471-2105-11-S4-P11

Cite this article as: Krushkal et al:: Bioinformatic analysis of gene regulation in the metal-reducing bacterial family Geobacteraceae. BMC Bioinformatics 2010 11(Suppl 4):P11.
* Correspondence: jkrushka@uthsc.edu

${ }^{1}$ Department of Preventive Medicine, University of Tennessee Health Science Center, Memphis, TN 38163, USA 